\title{
A COUNTEREXAMPLE FOR CS-RINGS
}

\author{
GARY F. BIRKENMEIER \\ Department of Mathematics, University of Louisiana, Lafayette, Louisiana 70504, U.S.A. \\ e-mail:gfb1127@usl.edu \\ JIN YONG KIM \\ Department of Mathematics, Kyung Hee University, Suwon 449-701, South Korea \\ e-mail:jykim@nms.kyunghee.ac.kr \\ and JAE KEOL PARK \\ Department of Mathematics, Busan National University, Busan 609-735, South Korea \\ e-mail:jkpark@hyowon.cc.pusan.ac.kr \\ Dedicated to Professor Klaus W. Roggenkamp on his sixtieth birthday
}

(Received 31 August, 1998)

\begin{abstract}
A module $M$ is called a $C S$-module or an extending module if every submodule is essential in a direct summand of $M$. A ring $R$ is called a right $C S$-ring or a right extending ring if $R_{R}$ is a CS-module. For several types of right CS-rings it is known that either all right ideals or some large class of right ideals inherit the CS property. For example, by a result of Dung-Smith or Vanaja-Purav, a ring $R$ is (right and left) Artinian, serial, and $J(R)^{2}=0$ if and only if every $R$-module is CS. In particular, if $R$ is a QF-ring and $J(R)^{2}=0$ (hence $R$ is serial), then every $R$-module is CS. However we exhibit a finite, serial, strongly bounded QF group algebra $R$ with $J(R)^{3}=0$ for which there is a principal right ideal which is a right essential extension of a CS-module and essential in $R_{R}$ but not CS itself.
\end{abstract}

1991 Mathematics Subject Classification. Primary 16D80; secondary 16D50.

Throughout this paper $R$ will denote an associative ring with unity, $J(R)$ is its Jacobson radical, and all modules will be unital right $R$-modules (unless otherwise indicated). A submodule $N$ of a module $M$ is called a closed submodule if there is no proper essential extension of $N$ inside $M$. A module $M$ is called a $C S$-module or an extending module if every submodule of $M$ is essential in a direct summand of $M$. Thus a module $M$ is CS if and only if every closed submodule of $M$ is a direct summand of $M$. The class of CS-modules includes all injective modules, all quasicontinuous modules, and all uniform modules. A ring $R$ is called right (resp. left) $C S$ or extending if $R_{R}$ (resp. ${ }_{R} R$ ) is CS or extending. The usefulness of the CS concept is well documented in [10] or more recently [3].

A module is called uniserial if it has a unique composition series of finite length. A ring $R$ is called right (resp. left) serial if $R_{R}\left(\operatorname{resp} .{ }_{R} R\right)$ is a finite direct sum of uniserial right (resp. left) ideals. A ring $R$ is called serial if it is both right and left serial.

Recall that a ring $R$ is called quasi-Frobenius (or simply QF) if $R$ is right or left Artinian and right or left self-injective. For a QF-ring $R$, it is well known that the right socle, $\operatorname{Soc}\left(R_{R}\right)$, of $R$ is equal to the left $\operatorname{socle,~} \operatorname{Soc}\left({ }_{R} R\right)$, of $R$. Without ambiguity, when $R$ is a QF-ring, we simply denote the right or left socle of $R$ by $\operatorname{Soc}(R)$. Also note that if a ring $R$ is QF, then $\ell(J(R))=r(J(R))=\operatorname{Soc}(R)$ and $\ell(\operatorname{Soc}(R))=r(\operatorname{Soc}(R))=J(R)$, where $J(R), \ell(-)$, and $r(-)$ are the Jacobson radical of $R$, the left annihilator, and the right annihilator, respectively. 
A ring $R$ is called strongly right (resp. left) bounded if every nonzero right (resp. left) ideal of $R$ contains a nonzero ideal. A ring $R$ is called strongly bounded if $R$ is both strongly right and strongly left bounded. Observe that if $R$ is strongly right bounded then every nonzero right ideal of $R$ is an essential extension of an ideal of $R$.

Unfortunately, the class of CS-modules is somewhat pathological in that it is not closed under homomorphic images, finite direct sums, or extensions. We shall show that this pathology extends to closure with respect to submodules. When investigating a class $\mathscr{N}$ of modules it is natural to ask: for $M \in \mathscr{A}$ under what conditions are the submodules (or some distinguished set of submodules) of $M$ also in $\Re$ ? In particular, we ask: if $R_{R}$ is CS, when are all right ideals (or some distinguished set of right ideals) of $R$ also CS? The following results provide some answers to this question.

(1) [3, p.134]. If $R_{R}$ is nonsingular and (finitely, countably) $\Sigma$-extending, then every (finitely, countably generated) right ideal is $C S$.

(2) $[4,13]$. Every module is $C S$ if and only if $R$ is (right and left) Artinian, serial, and $J(R)^{2}=0$. From $[\mathbf{6}, 25.4 .3]$, if $R$ is a $Q F$-ring with $J(R)^{2}=0$, then $R$ is serial. Thus in this case every $R$-module is $C S$.

(3) [1, Corollaries 1.3 and 2.2]. Let $R_{R}$ be CS. We have the following:

(i) every ideal is $C S$;

(ii) if every idempotent is central, then every right ideal is $C S$;

(iii) if $R_{R}$ is nonsingular, then every principal right ideal is $C S$.

A serial QF-ring $R$ with $J(R) \neq 0$ can arguably be considered a quintessential example of a CS-module which is not nonsingular. Observe that a QF-ring is $\Sigma$ injective and $\Sigma$-extending [3, p.95 and p.170]. From the above results one would expect that the CS condition will be inherited by a large class of right ideals of a serial QF-ring. In particular, in light of (1), (2), and (3), one could reasonably conjecture that if $R$ is a stongly bounded, PI, serial $Q F$-ring with $J(R)^{3}=0$, then every principal right ideal is CS. Surprisingly, this is not the case as is illustrated in the following example of a finite group algebra.

Not only is the choice of this group algebra counterintuitive but the calculations involve a degree of judgement (e.g., in Step 3 of Claim 4 there are three choices for the appropriate injective hull of $(2+\tau) R \cap(1+\sigma+\tau) R$ in the group algebra $R)$.

ExAmple. We consider the group algebra $R=\mathbb{Z}_{3}\left[S_{3}\right]$ of the symmetric group $S_{3}$ on three symbols $\{1,2,3\}$ over the field $\mathbb{Z}_{3}$ of three elements. Denote $\sigma=(123)$ and $\tau=(12)$ in $S_{3}$.

Note that the ring $R$ is right self-injective by [11, Theorem 2.8, p.79] and so $R$ is QF. Since $R$ is a finite ring, it is a PI-ring. We shall show that $R$ is serial, strongly bounded, and $J(R)^{3}=0$ such that there exists a principal right ideal of $R$ that is a right essential extension of a CS-module and it is essential in $R_{R}$, but it is not CS itself.

CLAIM 1. $J(R)^{3}=0$ and $R$ is strongly bounded.

Proof. By [11, Exercise 8, p.106] $J(R)$ is $\omega\left(\mathbb{Z}_{3}[N]\right) R$, where

$$
\omega\left(\mathbb{Z}_{3}[N]\right)=\left\{a+b \sigma+c \sigma^{2} \mid a+b+c=0, a, b, c \in \mathbb{Z}_{3}\right\},
$$


which is the augmentation ideal of $\mathbb{Z}_{3}[N]$ and $N=\langle\sigma\rangle$. Thus by direct calculation $J(R)=(2+\sigma) R$, which is

$$
\left\{a_{0}+a_{1} \sigma+2\left(a_{0}+a_{1}\right) \sigma^{2}+b_{0} \tau+b_{1} \sigma \tau+2\left(b_{0}+b_{1}\right) \sigma^{2} \tau \mid a_{0}, a_{1}, b_{0}, b_{1} \in \mathbb{Z}_{3}\right\} .
$$

Hence it can be easily checked that $J(R)^{3}=0$.

The vector space dimension of $J(R)$ over the field $\mathbb{Z}_{3}$ is four, and so the vector space dimension of $R / J(R)$ over $\mathbb{Z}_{3}$ is two. Therefore the number of elements of the $\operatorname{ring} R / J(R)$ is nine. Since $R$ is QF, the $\operatorname{ring} R / J(R)$ is semisimple Artinian. Note that the ring $R$ is not a local ring because there is a nontrivial idempotent in $R$, for example $2+\tau$. Hence, by Wedderburn-Artin, $R / J(R) \cong \mathbb{Z}_{3} \bigoplus \mathbb{Z}_{3}$ as rings. Thus $R$ is basic. Now, by [7, Theorem $1.7 \mathrm{~B}], R$ is a strongly bounded ring.

According to [2, Definition 7.11, p.480], recall that an algebra $A$ over a field $F$ is called a separable algebra over $F$ if $A \bigotimes_{F} H$ is a semisimple algebra over $H$, for every extension field $H$ of $F$. Following [12], for a prime number $p$, recall that a group is called $p$-solvable if each of its composition factors is either a $p$-group or has order prime to $p$.

\section{Claim 2. $R$ is a serial ring.}

Proof. Now in our situation, since $R / J(R) \cong \mathbb{Z}_{3} \oplus \mathbb{Z}_{3}$, it follows that the $\mathbb{Z}_{3}$ algebra $R / J(R)$ is separable over the field $\mathbb{Z}_{3}$. Let $\overline{\mathbb{Z}}_{3}$ be the algebraic closure of the field $\mathbb{Z}_{3}$. Then the group ring $\overline{\mathbb{Z}}_{3}\left[S_{3}\right]=\overline{\mathbb{Z}}_{3} \bigotimes_{\mathbb{Z}_{3}} R$ is serial by [12, Theorem 3] because the group $S_{3}$ is 3 -solvable with a 3-Sylow subgroup. Since $R / J(R)$ is separable over $\mathbb{Z}_{3}$, the ring $R$ is serial by [5, Theorem 4.1$]$.

REMARK 3. Explicitly, by direct calculation, $(2+\tau) R$ has the unique composition series

$$
0 \subsetneq \operatorname{Soc}((2+\tau) R) \subsetneq(2+\tau) R \cap(1+\sigma+\tau) R \subsetneq(2+\tau) R
$$

and also $(2+2 \tau) R$ has the unique composition series

$$
0 \subsetneq \operatorname{Soc}((2+2 \tau) R) \subsetneq(2+2 \tau) R \cap(1+\sigma+\tau) R \subsetneq(2+2 \tau) R .
$$

Thus $R=(2+\tau) R \bigoplus(2+2 \tau) R$ is right serial. Similarly, $R=R(2+\tau) \bigoplus R(2+2 \tau)$ is left serial. Consequently, $R$ is a serial ring.

CLAIM 4. There is a principal right ideal of $R$ that is a right essential extension of a CS-module and essential in $R_{R}$, but it is not CS itself. In fact, we have that $(1+\sigma+\tau) R$ is such a principal right ideal.

STEP 1. The right uniform dimension of $R$ is two.

$$
\begin{gathered}
\text { Proof of Step 1. } \operatorname{Soc}(R)=\ell(J(R))=\left(1+\sigma+\sigma^{2}\right) R \text { because } R \text { is QF. Hence } \\
\operatorname{Soc}(R)=\left\{a\left(1+\sigma+\sigma^{2}\right)+b\left(1+\sigma+\sigma^{2}\right) \tau \mid a, b \in \mathbb{Z}_{3}\right\} .
\end{gathered}
$$

Thus the vector space dimension of $\operatorname{Soc}(R)$ over the field $\mathbb{Z}_{3}$ is two. Since $R$ has a nontrivial idempotent, the right uniform dimension of $\operatorname{Soc}(R)$ is greater than one. 
Therefore the right uniform dimension of $R$ is two because $\operatorname{Soc}(R)$ is an essential $R$ submodule of $R_{R}$.

STEP 2. The form of elements from $(2+\tau) R \cap(1+\sigma+\tau) R$ is

$$
a+b \sigma+(2 a+2 b) \sigma^{2}+2 a \tau+(a+b) \sigma \tau+2 b \sigma^{2} \tau,
$$

for some $a, b \in \mathbb{Z}_{3}$.

Proof of Step 2. Note that

$$
\begin{aligned}
(1+\sigma+\tau) R & =\left\{\left(a_{0}+a_{2}+b_{0}\right)+\left(a_{0}+a_{1}+b_{2}\right) \sigma+\left(a_{1}+a_{2}+b_{1}\right) \sigma^{2}+\left(a_{0}+b_{0}+b_{2}\right) \tau\right. \\
& \left.+\left(a_{2}+b_{0}+b_{1}\right) \sigma \tau+\left(a_{1}+b_{1}+b_{2}\right) \sigma^{2} \tau \mid a_{i}, b_{i} \in \mathbb{Z}_{3}, i=0,1,2\right\}
\end{aligned}
$$

and

$$
(2+\tau) R=\left\{a+b \sigma+c \sigma^{2}+2 a \tau+2 c \sigma \tau+2 b \sigma^{2} \tau \mid a, b, c \in \mathbb{Z}_{3}\right\} .
$$

Direct calculation yields the desired form of elements in $(2+\tau) R \cap(1+\sigma+\tau) R$.

STEP 3. $(2+\tau) R \cap(1+\sigma+\tau) R$ has no proper essential extension in $(1+\sigma+\tau) R$. Moreover, $(2+\tau) R \cap(1+\sigma+\tau) R$ has a unique injective hull in $R_{R}$.

Proof of Step 3. For our convenience, let $f=2+\tau$. Let $K$ be a maximal essential extension of $f R \cap(1+\sigma+\tau) R$ in $(1+\sigma+\tau) R$. Then there is an injective hull $E(f R \cap(1+\sigma+\tau) R)=g R$ of $f R \cap(1+\sigma+\tau) R$ with $g=g^{2} \in R$ such that $K \subseteq g R$ and so $K \subseteq g R \cap(1+\sigma+\tau) R$. Since $f R \cap(1+\sigma+\tau) R$ is essential in $g R \cap(1+\sigma+\tau) R$, it follows that $K=g R \cap(1+\sigma+\tau) R$. Furthermore since $g R$ is an injective hull of $f R \cap(1+\sigma+\tau) R$, we have that $f R \cong g R$.

Since $f R \cap(1+\sigma+\tau) R$ is essential in $K$ and $(f R \cap(1+\sigma+\tau) R) \cap(1-f) R=0$, it follows that $K \cap(1-f) R=0$. Also since $K$ is essential in $g R$ and $K \cap(1-f) R=0$, we have that $g R \cap(1-f) R=0$. Since $g R \cap(1-f) R=0$ and, by Step 1, the right uniform dimension of $R$ is two, it follows that $R=g R \bigoplus(1-f) R$. We claim that

$$
\bar{R}=\bar{g} \bar{R} \bigoplus(\overline{1}-\bar{f}) \bar{R},
$$

where $\bar{R}=R / J(R)$. Obviously $\bar{R}=\bar{g} \bar{R}+(\overline{1}-\bar{f}) \bar{R}$. Now if $\bar{g} \bar{R} \cap(\overline{1}-\bar{f}) \bar{R} \neq \overline{0}$, then, since $\bar{R} \cong \mathbb{Z}_{3} \bigoplus \mathbb{Z}_{3}, \bar{g} \bar{R} \cap(\overline{1}-\bar{f}) \bar{R}$ is a minimal ideal of $\bar{R}$. If the uniform dimension of $\bar{g} \bar{R}$ is two, then $\bar{g} \bar{R}=\bar{R}$ and so $\bar{g}$ is invertible and hence $g$ is invertible in $R$, which is a contradiction. Thus the uniform dimension of $\bar{g} \bar{R}$ is one, and so $\bar{g} \bar{R}$ is a minimal right ideal of $\bar{R}$. Thus $\bar{g} \bar{R} \cap(\overline{1}-\bar{f}) \bar{R}=\overline{0}$, so that

$$
\bar{R}=\bar{g} \bar{R} \bigoplus(\overline{1}-\bar{f}) \bar{R}=\bar{f} \bar{R} \bigoplus(\overline{1}-\bar{f}) \bar{R} .
$$

Since $\bar{R}$ is commutative, $\bar{g} \bar{f}$ is an idempotent. If $\bar{g} \bar{f}=\overline{0}$, then $\bar{g} \bar{R} \subseteq(\overline{1}-\bar{f}) \bar{R}$. Thus $\bar{g} \bar{f} \neq \overline{0}$. Also $\bar{g} \bar{f} \bar{R} \subseteq \bar{f} \bar{R}$ and $\bar{g} \bar{f} \bar{R} \subseteq \bar{g} \bar{R}$. But both $\bar{f} \bar{R}$ and $\bar{g} \bar{R}$ have the vector space dimension one over $\mathbb{Z}_{3}$. Thus $\bar{f} \bar{R}=\bar{g} \bar{f} \bar{R}=\bar{g} \bar{R}$. Consequently, since $\bar{R}$ is commutative, $\bar{f}=\bar{g}$ and so $g=f+\gamma$, for some $\gamma \in J(R)=(2+\sigma) R$.

Therefore in the proof of Claim 1 , 


$$
\gamma=a_{0}+a_{1} \sigma+2\left(a_{0}+a_{1}\right) \sigma^{2}+b_{0} \tau+b_{1} \sigma \tau+2\left(b_{0}+b_{1}\right) \sigma^{2} \tau,
$$

for some $a_{i}, b_{i} \in \mathbb{Z}_{3}, i=0,1$; hence we have

$$
g=\left(2+a_{0}\right)+a_{1} \sigma+2\left(a_{0}+a_{1}\right) \sigma^{2}+\left(1+b_{0}\right) \tau+b_{1} \sigma \tau+2\left(b_{0}+b_{1}\right) \sigma^{2} \tau .
$$

We now compute the coefficients of $g^{2}$ and compare them with those of $g$.

(i) The coefficient of 1 in $g^{2}$ is

$$
\left(2+a_{0}\right)^{2}+2 a_{1}\left(a_{0}+a_{1}\right)+2\left(a_{0}+a_{1}\right) a_{1}+\left(1+b_{0}\right)^{2}+b_{1}^{2}+4\left(b_{0}+b_{1}\right)^{2},
$$

which is equal to $2+a_{0}$. Thus we have the relation

$$
a_{0}^{2}+a_{0} a_{1}+a_{1}^{2}+2 b_{0}^{2}+2 b_{0} b_{1}+2 b_{1}^{2}+2 b_{0}=0 .
$$

(ii) The coefficient of $\tau$ in $g^{2}$ is

$$
\begin{aligned}
& \left(2+a_{0}\right)\left(1+b_{0}\right)+2 a_{1}\left(b_{0}+b_{1}\right)+2\left(a_{0}+a_{1}\right) b_{1} \\
& +\left(1+b_{0}\right)\left(2+a_{0}\right)+a_{1} b_{1}+4\left(a_{0}+a_{1}\right)\left(b_{0}+b_{1}\right),
\end{aligned}
$$

which is equal to $1+b_{0}$. It follows that $2 a_{0}=0$ and so $a_{0}=0$.

(iii) The coefficient of $\sigma \tau$ in $g^{2}$ is

$$
\begin{aligned}
& \left(2+a_{0}\right) b_{1}+a_{1}\left(1+b_{0}\right)+4\left(a_{0}+a_{1}\right)\left(b_{0}+b_{1}\right) \\
& +2\left(b_{0}+b_{1}\right)\left(a_{0}+a_{1}\right)+b_{1}\left(2+a_{0}\right)+2\left(b_{0}+b_{1}\right) a_{1},
\end{aligned}
$$

which is equal to $b_{1}$. Thus $a_{0}+2 a_{1}=0$. Since $a_{0}=0$, we have that $a_{1}=0$.

From (i), (ii) and (iii), it follows that $2 b_{0}^{2}+2 b_{0} b_{1}+2 b_{1}^{2}+2 b_{0}=0$, and so

$$
b_{0}^{2}+b_{0} b_{1}+b_{1}^{2}+b_{0}=0 .
$$

Thus there are only three possibilities for $b_{0}$ and $b_{1}: b_{0}=b_{1}=0 ; b_{0}=2, b_{1}=0$; and $b_{0}=2, b_{1}=1$. Therefore $g=f, g=2+\sigma \tau$ or $g=2+\sigma^{2} \tau$. Hence all candidates for maximal essential extensions of $(2+\tau) R \cap(1+\sigma+\tau) R$ in $(1+\sigma+\tau) R$ are $(2+\tau) R \cap(1+\sigma+\tau) R,(2+\sigma \tau) R \cap(1+\sigma+\tau) R$, and $\left(2+\sigma^{2} \tau\right) R \cap(1+\sigma+\tau) R$.

Note that $1+2 \sigma+2 \tau+\sigma^{2} \tau=(2+\tau)(2+\sigma)=(1+\sigma+\tau)\left(1+\sigma+2 \sigma^{2}+\tau\right)$, and so we have $1+2 \sigma+2 \tau+\sigma^{2} \tau \in(2+\tau) R \cap(1+\sigma+\tau) R$. However $1+2 \sigma+2 \tau+\sigma^{2} \tau \notin(2+\sigma \tau) R$ and $1+2 \sigma+2 \tau+\sigma^{2} \tau \notin\left(2+\sigma^{2} \tau\right) R$. Therefore it follows that $(2+\tau) R \cap(1+\sigma+\tau) R \nsubseteq(2+\sigma \tau) R$ and $(2+\tau) R \cap(1+\sigma+\tau) R$ $\nsubseteq\left(2+\sigma^{2} \tau\right) R$. Consequently, $(2+\tau) R \cap(1+\sigma+\tau) R$ is a closed submodule in $(1+\sigma+\tau) R$. Also $(2+\tau) R$ is the unique injective hull of $(2+\tau) R \cap(1+\sigma+\tau) R$ in $R_{R}$.

STEP 4. $(1+\sigma+\tau) R$ is not CS as a right $R$-module.

Proof of Step 4. Assume to the contrary that $(1+\sigma+\tau) R$ is CS as a right $R$ module. Then, since $(2+\tau) R \cap(1+\sigma+\tau) R$ is a closed submodule in $(1+\sigma+\tau) R$ 
by Step 3, it is a direct summand of $(1+\sigma+\tau) R$; and so we have a projection $\pi$ from $(1+\sigma+\tau) R$ onto $(2+\tau) R \cap(1+\sigma+\tau) R$. Since $R$ is right self-injective, there is an $R$-homomorphism $\theta$ from $R$ to $R$ that extends the $R$-homomorphism $i \circ \pi$, where $i$ is the inclusion from $(2+\tau) R \cap(1+\sigma+\tau) R$ to $R$. Hence $(i \circ \pi)(1+\sigma+\tau)=\pi(1+\sigma+\tau)=\theta(1+\sigma+\tau)=\theta(1)(1+\sigma+\tau)=x_{0}(1+\sigma+\tau)$, where $x_{0}=\theta(1) \in R$. Thus we have in this case

$$
x_{0}(1+\sigma+\tau) \in(2+\tau) R \cap(1+\sigma+\tau) R .
$$

Say $x_{0}=a_{0}+a_{1} \sigma+a_{2} \sigma^{2}+b_{0} \tau+b_{1} \sigma \tau+b_{2} \sigma^{2} \tau$, for some $a_{i}, b_{i} \in \mathbb{Z}_{3}$ for $i=0,1,2$. Then $\quad\left(a_{0}+a_{1} \sigma+a_{2} \sigma^{2}+b_{0} \tau+b_{1} \sigma \tau+b_{2} \sigma^{2} \tau\right)(1+\sigma+\tau)$ $=\left(a_{0}+a_{2}+b_{0}\right)+\left(a_{0}+a_{1}+b_{1}\right) \sigma+\left(a_{1}+a_{2}+b_{2}\right) \sigma^{2}+\left(a_{0}+b_{0}+b_{1}\right) \tau$ $+\left(a_{1}+b_{1}+b_{2}\right) \sigma \tau+\left(a_{2}+b_{0}+b_{2}\right) \sigma^{2} \tau$ is in $(2+\tau) R \cap(1+\sigma+\tau) R$. Since

$(2+\tau) R \cap(1+\sigma+\tau) R \subseteq\left\{a+b \sigma+(2 a+2 b) \sigma^{2}+2 a \tau+(a+b) \sigma \tau+2 b \sigma^{2} \tau \mid a, b \in \mathbb{Z}_{3}\right\}$

by Step 2, we have that

$$
\begin{gathered}
a_{0}+a_{2}+b_{0}=a, \\
a_{0}+a_{1}+b_{1}=b, \\
a_{1}+a_{2}+b_{2}=2 a+2 b
\end{gathered}
$$

and

$$
\begin{gathered}
a_{0}+b_{0}+b_{1}=2 a, \\
a_{1}+b_{1}+b_{2}=a+b, \\
a_{2}+b_{0}+b_{2}=2 b,
\end{gathered}
$$

for some $a, b \in \mathbb{Z}_{3}$.

From the equations $a_{0}+b_{0}+b_{1}=2 a, \quad a_{1}+b_{1}+b_{2}=a+b, \quad$ and $a_{2}+b_{0}+b_{2}=2 b$ in (2), we have $a_{0}=2 a+2 b_{0}+2 b_{1}, a_{1}=a+b+2 b_{1}+2 b_{2}$, and $a_{2}=2 b+2 b_{0}+2 b_{2}$, respectively. By substituting these equations into (1), we get that $2 b_{0}+2 b_{1}+2 b_{2}=2 a+b, b_{0}+b_{1}+b_{2}=0, \quad$ and $2 b_{0}+2 b_{1}+2 b_{2}=a+2 b$. Thus we have that $a+2 b=0$, or equivalently, $a=b$. Therefore, it follows that

$x_{0}(1+\sigma+\tau)=a+a \sigma+a \sigma^{2}+2 a \tau+2 a \sigma \tau+2 a \sigma^{2} \tau=a\left(1+\sigma+\sigma^{2}\right)+2 a\left(1+\sigma+\sigma^{2}\right) \tau$

and so it is in $\left(1+\sigma+\sigma^{2}\right) R$. Thus $\pi(1+\sigma+\tau) \in\left(1+\sigma+\sigma^{2}\right) R$, and hence

$$
(2+\tau) R \cap(1+\sigma+\tau) R=\pi(1+\sigma+\tau) R \subseteq\left(1+\sigma+\sigma^{2}\right) R .
$$

Finally as we previously noted, $1+2 \sigma+2 \tau+\sigma^{2} \tau \in(2+\tau) R \cap(1+\sigma+\tau) R$. However $1+2 \sigma+2 \tau+\sigma^{2} \tau \notin\left(1+\sigma+\sigma^{2}\right) R$, and so we have a contradiction. Consequently, $(1+\sigma+\tau) R$ is not $\mathrm{CS}$ as a right $R$-module.

STEP 5. There exists an ideal I of $R$ such that $I$ is right $C S$ and right essential in $(1+\sigma+\tau) R$ and $(1+\sigma+\tau) R$ is essential in $R_{R}$.

Proof of Step 5. By Claim 1, since $R$ is strongly bounded, there exsits an ideal $I$ of $R$ such that $I \subseteq(1+\sigma+\tau) R$ and $I$ is right essential in $(1+\sigma+\tau) R$. In this situation, since $R_{R}$ is $\mathrm{CS}, I$ is $\mathrm{CS}$ by $[\mathbf{1}$, Corollary 1.3 (ii)]. Since 
$\operatorname{Soc}((2+\tau) R) \bigoplus \operatorname{Soc}((2+2 \tau) R) \subseteq(1+\sigma+\tau) R$ by Remark 3 and the right uniform dimension of $R$ is two by Step 1 , the uniform dimension of $(1+\sigma+\tau) R$ is two. Hence $(1+\sigma+\tau) R$ is essential in $R_{R}$.

REMARK 5. It is well known that an Artinian CS-module is a finite direct sum of uniform modules. But, in the proof of Example, we can show that the converse does not hold even for a finite QF-ring. Since $2+\sigma^{2} \tau \in(1+\sigma+\tau) R$ and $2+\sigma^{2} \tau$ is an idempotent, $(1+\sigma+\tau) R=\left(2+\sigma^{2} \tau\right) R \bigoplus Y$, for some submodule $Y$ of $(1+\sigma+\tau) R$. Since $\left(2+\sigma^{2} \tau\right) R$ is injective and uniform, and by Steps 1 and 5 of Claim 4 the uniform dimension of $(1+\sigma+\tau) R$ is two, it follows that $Y$ is also uniform. Hence the direct sum of two Artinian uniform modules is not necessarily CS. For more on finite direct sums of CS-modules, see [8]. However in [9] it was proved that if $R$ is a QF-ring which is serial with $J(R)^{3}=0$, then every right $R$-module is a direct sum of a projective module and a CS-module. It follows that every principal right ideal of $\mathbb{Z}_{3}\left[S_{3}\right]$ is a direct sum of an injective module and a CS-module.

AcKnowledgements. The first author is grateful for the gracious hospitality he received at Busan National University and Kyung Hee University. The second author was supported in part by KOSEF Research Grant 97-k3-0101 (RCAA) and the Basic Science Research Institute Program, Ministry of Education, Korea in 1997, Project No.BSRI-97-1432, while the third author was partially supported by KOSEF, Project No.971-0101-005-2 and the Basic Science Research Institute Program, Ministry of Education, Korea in 1997, Project No.BSRI-97-1402.

\section{REFERENCES}

1. G. F. Birkenmeier, J. Y. Kim and J. K. Park, When is the CS condition hereditary?, Comm. Algebra 27 (1999), 3875-3885.

2. C. W. Curtis and I. Reiner, Representation theory of finite groups and associative algebras (Interscience, New York, 1962).

3. N. V. Dung, D. V. Huynh, P. F. Smith and R. Wisbauer, Extending modules (Pitman, London, 1994).

4. N. V. Dung and P. F. Smith, Rings for which certain modules are CS, J. Pure Appl. Algebra 102 (1995), 273-287.

5. D. Eisenbud and P. Griffith, Serial rings, J. Algebra 17 (1971), 389-400.

6. C. Faith, Algebra II-Ring theory (Springer-Verlag, 1976).

7. C. Faith and S. Page, FPF Ring theory: Faithful modules and generators of Mod-R, London Math. Soc. Lecture Note Series, Vol. 88 (Cambridge University Press, 1984).

8. A. Harmanci and P. F. Smith, Finite direct sums of CS-modules, Houston J. Math. 19 (1993), 523-532.

9. D. V. Huynh and S. T. Rizvi, On some classes of artinian rings, J. Algebra 233 (2000), 133-153.

10. S. H. Mohamed and B. J. Müller, Continuous and discrete modules, London Math. Soc. Lecture Note 147 (Cambridge University Press, 1990).

11. D. S. Passman, The algebraic structure of group rings (John Wiley, New York, 1977).

12. B. Srinivasan, On the indecomposable representation of a certain class of groups, Proc. London Math. Soc. (3) 10 (1960), 497-513.

13. N. Vanaja and V. M. Purav, Characterizations of generalized uniserial rings in terms of factor rings, Comm. Algebra 20 (1992), 2253-2270. 